\title{
ORGANIZACIÓN Y PROMOCIÓN DE LAS ACTIVIDADES ARTÍSTICAS EN CIUDAD JUÁREZ, CHIHUAHUA (1945-1990)
}

\author{
Por \\ César Fuentes Flores* \\ Jesús Montenegro Herrera*
}

\begin{abstract}
RESUMEN
En este trabajo se analiza la manera en que se ha organizado la promoción, el consumo, así comoel nivel de institucionalización de las actividades artísticas en Ciudad Juárez durante el periodo 1945-1990. Asimismo, se reconstruye cómo y cuáles agentes sociales participaban en la promoción de las actividades artísticas y culturales, cuál era el esquema de promoción que seguían dichos grupos en cuanto al tipo de especialidades artísticas que organizaban, así como cuáles fueron los espacios físicos utilizados para el desarrollo de este tipo de actividades.

En general la información utilizada es hemerográfica, del mismo modo se realizaron entrevistas con informantes claves.
\end{abstract}

\begin{abstract}
In this work it is analized the way in which promotion and consumption have been organized, as well as the institucionalization of the artistic activities in Ciudad Juarez during the $1945-1990$ period. It also recontructs how and which social agents participated in the promotion scheme that such groups followed according to the type of artistic craft they organized, as well as which were the physical spaces used for the development of these types of activities.

At large, the information used was merely hemerographic, and in the same way, interviews were done with key informants.
\end{abstract}

\section{INTRODUCCIÓN}

Ciudad Juárez, como la mayor parte de las poblaciones fronterizas del norte de México, durante mucho tiempo fue considerada como "desierto cultural", en donde proliferaban los casinos, bares, etcétera, incluso algunos religiosos extremistas la calificaban como "la ciudad del pecado".

* Investigadores de El Colegio de la Frontera Norte.

1 "Desierto cultural" en el sentido de conjunto de carencias, lagunas, de necesidades no cubiertas en materia de "cultura culta" (véase León-Portilla, 1992). 
Las ciudades fronterizas también han sido consideradas como la frontera cultural, es decir, el límite entre la barbarie y la cultura, o resguardomuralla cultural frente a posibles "infiltraciones externas".

Desde el triunfo de la revolución, el Estado habría de ser el promotor y guardian de la cultura y de la "identidad nacional", lo cual fue posible debido al patrón de alianzas establecidas durante el cuatrienio de Obregón; entre Vasconcelos, los muralistas y el gobierno central.

Sin embargo, este patrón de alianzas no llego a todas las regiones del país, por lo que las ciudades de la frontera norte de México no formaron parte en los movimientos de institucionalización de la "cultura nacional", debido, principalmente, a su localización geográfica respecto al centro cultural del país y de las capitales de los estados, en donde se concentran los mayores apoyos económicos y humanos para la promoción de las actividades artísticas y culturales.

Como consecuencia, la organización, producción y consumo de las actividades artísticas muestra matices propios producto de su realidad fronteriza.

Bajo este panorama, el propósito del presente trabajo es dar respuesta a la pregunta principal: ¿Cómo se han organizado la promocion y el consumo artístico en Ciudad Juárez, durante el periodo 1945-1990?

\section{NOTA METODOLÓGICA}

Para reconstruir la organización y promoción de las actividades artísticas y culturales en Ciudad Juárez se utilizaron, básicamente, fuentes hemerográficas. ${ }^{3}$ Se llenaron formatos concentrando información sobre eventos artísticos ${ }^{4}$ con variables como: nombre del grupo o asociacion, año de su fundación, especialidad artística, tipo de evento, nombres de promotores y lugares, etcétera.

Se catalogaron como eventos artísticos todos aquellos relacionados con cualesquiera de las siguientes expresiones culturales: música "clásica", teatro (de cualquier género), literatura, danza (de cualquier tipo) y lo que actualmente se denominan "artes visuales" en cualquiera de sus formatos.

2 Esta idea de los límites culturales o límites espaciales de la cultura o civilización de la cristanidad formó parte esencial del mito de la cultura occidental (ver G. Mairet, 1978).

${ }^{3}$ Es importante aclarar que las fuentes hemerográficas tienen sus limitaciones como son inexactitud de numerosos datos, sesgos producidos por el periodista, traducción de hechos en noticias. Sin embargo, se reconocerá que difícilmente se puede acudir a otras fuentes menos sesgadas.

4 La fuente hemerográfica utilizada fue el periódico El Fronterizo, y se eligió éste debido a que es el más antiguo de los que actualmente existen y por lo tanto era el único que nos permitiría realizar la reconstrucción histórica. 
En general, se adoptó una definición de lo artístico en el sentido "socialmente reconocido como artístico". Lo anterior se realizó para cada quinquenio de 1945 a 1990. Estos años se agruparon por periodos, que se definieron por el nivel de institucionalización de las actividades artísticas; 1945-1960, 1965-1980 y 1985-1990. Asimismo, se formaron grupos dada la diversidad de asociaciones y organizaciones que participaron como promotores artísticos, estos se catalogaron de la siguiente manera: promotores ocasionales, ${ }^{5}$ individuales, ${ }^{6}$ asociaciones consumidoras de arte, ${ }^{7}$ productores, ${ }^{8}$ academias y el Estado en sus tres niveles de gobierno. También se realizaron entrevistas con informantes claves de los diferentes grupos y especialidades artísticas.

\section{LA PROMOCIÓN DE LAS ACTIVIDADES ARTÍSTICAS EN CIU- DAD JUÁREZ, CHIHUAHUA 1945-1990}

Existe cierto desconocimiento de cómo se han desarrollado las actividades artísticas y culturales en Ciudad Juárez, por lo que para reconstruir la organización y promoción de éstas, es necesario saber cómo se gestaron dichas actividades, lo cual nos permitirá advertir qué agentes sociales participaban en su organización.

En la revisión hemerográfica que se hizo sobre la promoción de las actividades artísticas se distinguen claramente tres periodos, 1945-1960, $1965-1980$ y 1985-1990, los cuales están definidos por su nivel de institucionalización.

\section{Periodo 1945-1960}

Durante el periodo 1945-1960, del total de eventos promovidos en Ciudad Juárez, el $40 \%$ correspondió a promotores ocasionales, el $33 \%$ a asociaciones consumidoras de arte, el $9 \%$ a promotores individuales, y el resto a otro tipo de grupos (ver cuadro 1).

\footnotetext{
5 Agrupamos como promotores ocasionales, a aquellos grupos que su función no era principalmente la promoción de las actividades artísticas o culturales, y que además su promoción era ocasional. Por ejemplo, los clubes de servicio, tiendas, escuelas, étcétera.

Agrupamos en esta categoría a las personas o grupos de personas que en forma independiente promovían las actividades artísticas o culturales. Por ejemplo: un músico, pintor, cantante.

En esta categoría se concentró a los grupos cuya actividad principal era la promoción de la cultura. Por ejemplo: Ateneo Fronterizo, Asociación de Conciertos de la Comunidad.

Se incluyen a los productores de arte, los cuales tenían que promover sus propios eventos. Por ejemplo: grupos o escuelas de pintores, grupos de teatro.
} 
CUADRO 1. Número de eventos artísticos según tipo de promotor y periodos en Ciudad Juárez, Chihuahua, 1945-1990 (porcentajes).

\begin{tabular}{lccc}
\hline $\begin{array}{l}\text { Tipo de } \\
\text { Promotores }\end{array}$ & 1945-1960 & $\begin{array}{c}\text { Periodos } \\
1965-1980\end{array}$ & $1985-1990$ \\
\hline Ocasionales & 40 & 19 & 10 \\
Individuales & 9 & 1 & \\
Productores & 2 & 3 & 3 \\
Consumidores & 33 & 14 & 3 \\
Academias & 7 & 3 & \\
Iglesia & 6 & 6 & 28 \\
Universidad & 1 & 8 & 14 \\
Municipio & 1 & 47 & 42 \\
Estado & & 100 & 100 \\
Federacion & 100 & $\mathrm{n}=103$ & $\mathrm{n}=104$ \\
Total & $\mathrm{n}=82$ & & \\
& & & \\
\hline
\end{tabular}

FUENTE: Proyecto "Espacios Artísticos, Ciudad Juárez", 1992, El Colegio de la Frontera Norte, Oficina Regional en Ciudad Juárez.

En este periodo, el papel que juegan los promotores ocasionales (logias masonicas, clubes de servicio social, medios de comunicación, empresas), es definitivo, ya que la mayor parte de los eventos artísticos estuvieron en sus manos. Estos grupos organizaban programas culturales ante el vacío institucional que existía en materia de promoción artística. Sin embargo, la mayor parte de sus eventos eran esporádicos y no tenían coordinación entre las diferentes agrupaciones sociales, por lo que su impacto y continuidad era poco efectiva.

\section{a) Promotores ocasionales}

$\mathrm{Al}$ analizar en forma independiente cada uno de los años del periodo, encontramos que entre 1945 y 1950 el papel que juegan los grupos de promotores ocasionales es definitivo, ya que en 1945 organizaron el $38 \%$ de las actividades artísticas, en 1950 incrementaron su participación en un $41.1 \%$, y lo mismo ocurre durante 1955 . 
Entre los grupos que tuvieron una mayor presencia durante estos años se encuentran el Club de Leones, Club Deportivo Juvenil, Club Sorosis, Logia Sucursal Paso del Norte, Club 20-30, estación de radio XEWG, entre otros.

Sin embargo, para 1960, la participacion de los promotores ocasionales fue de $35.7 \%$, es decir, disminuyó en comparación con los años anteriores. En contraposición el Estado, en su nivel municipal, aumenta su presencia a un $14.2 \%$ y lo mismo ocurre con los grupos artísticos independientes.

\section{b) Asociaciones consumidoras de arte}

Por su parte, en este mismo periodo, las asociaciones consumidoras de arte generaron el $33 \%$ de los eventos artísticos. Dentro de estos grupos de promotores sobresale el Ateneo Fronterizo, ${ }^{9}$ la Sociedad Manuel M. Ponce y la Asociación de Conciertos de la Comunidad de Juárez; organizaciones que le dieron mayor impulso al quehacer artístico en Ciudad Juárez.

El Ateneo Fronterizo desde tiempo atrás sobresalió como uno de los pocos organismos interesados en la difusión de las actividades artísticas en la ciudad; uno de sus fundadores menciona:

...Cuando en los años treinta, Ciudad Juárez era blanco de las críticas por la proliferación de los casinos y los bares y algunos religiosos extremistas la tildaban de la "nueva Sodoma", "la ciudad del pecado" y aseguraban que todos sus habitantes se encontraban atrapados por la "lujuria", surgió un grupo cultural conformado por diversos profesionistas que se dio a la tarea de borrar esa imagen que se proyectaba hasta el interior del país... (Entrevista con el Sr. José María Mercado). ${ }^{10}$

Tan importante fue la promoción artística hecha por el Ateneo Fronterizo, que si analizamos al interior de este periodo, encontramos que este grupo sobresale en 1945 con un $25 \%$ de los eventos artísticos promovidos y en 1950 con el $23.5 \%$.

En 1955, los grupos antes mencionados generaron el $20.7 \%$ de los eventos artísticos, sobresaliendo en este año la Asociación de Conciertos de la Comunidad de Ciudad Juárez, la cual mantiene el mismo nivel de promoción para 1960. Es importante mencionar que esta asociación tenía su contraparte en El Paso Texas, por lo que generalmente los programas

\footnotetext{
9 El Ateneo Fronterizo nació el 23 de marzo de 1932, y es uno de los grupos culturales más antiguos de la ciudad si no es que el único. Fue fundado por Víctores Prieto, Manuel Ayala, Manuel Gómez Lomelí, Ricardo Carrillo Durán, Manuel L. Cardona, Esteban Briones, José Suárez y Heriberto García.

${ }_{10}$ Fragmento obtenido de la entrevista con el Sr. José María Mercado, uno de los más antiguos miembros del Ateneo Fronterizo.
} 
artísticos se presentaban en ambas ciudades. Este elemento de vecindad internacional entre dos ciudades de países con maneras distintas de promover el "arte", permitio que en esa época en Ciudad Juárez se llegaran a conocer a grandes personalidades del arte a nivel mundial. ${ }^{11}$

En este mismo año hace su incursión el Estado, en sus niveles municipal y estatal, con una participación del $4.1 \%$, la cual es muy baja en comparacion con los promotores ocasionales y las asociaciones abiertamente dedicadas a la organización de actividades artísticas.

Estos grupos promotores se caracterizan porque mantienen una alta independencia del Estado en sus activades, y además, son agrupaciones formadas por "amantes del arte", es decir, por consumidores no necesariamente especializados.

Cabe destacar durante el periodo 1945-1960, la total ausencia de los organismos oficiales en la promocion artística y cultural, por lo que grupos de profesionistas de clase media ven la necesidad de organizar eventos artísticos con sus propios recursos, con el propósito de borrar la mala imagen que se tenía de la ciudad, además de "alimentar el espíritu" con estas nobles manifestaciones de arte.

Lo anterior queda de manifiesto en la entrevista realizada a un miembro del Ateneo Fronterizo que declara:

El Ateneo Fronterizo desde su fundación era sostenido sólo por las aportaciones monetarias de sus propios socios, las cuales eran de dos pesos mensuales, aunque para 1940 era una cantidad considerable, no alcanzaba a cubrir los gastos de la asociación y las autoridades municipales no estaban interesadas en promover la cultura, ya que era más redituable el tipo de turismo que imperaba en aquellos días.

En suma, durante este periodo las prácticas artísticas se encontraban en un nivel de baja institucionalización. La continuidad del arte estaba solamente dada por las asociaciones de promotores que sobrevivieron durante esos años con recursos propios, y que se caracterizaron por su volatilidad institucional o su débil continuidad en este tipo de actividades.

\section{Periodo 1965-1980}

De 1965 a 1980 , del total de las actividades promovidas, el $47 \%$ le correspondio al gobierno federal, el $19 \%$ a promotores ocasionales, el $14 \%$

\footnotetext{
It Entre los artistas que se presentaron en el Ateneo Fronterizo se encuentran el violinista Jaime Laredo, la cantante Fania Nitúa, el bailarín Yuri Menguin, entre otros.
} 
a asociaciones consumidoras de arte, el $20 \%$ forma parte de otros grupos (véase cuadro 1).

En estos años resalta de manera importante la participación del gobierno federal como organizador. Por su parte, los promotores ocasionales y las asociaciones consumidoras de arte disminuyeron su participación relativa de acuerdo al periodo anterior, sin embargo, se siguen manteniendo como importantes grupos impulsores de las actividades artísticas.

\section{a) Promotores ocasionales}

Al hacer una revisión de este periodo, encontramos que en 1965 los promotores ocasionales incrementan su presencia en relacion con el quinquenio anterior, al alcanzar el $64 \%$. Es importante mencionar que hasta este año la organización continúa en manos de estos grupos de promotores como clubes sociales, clubes deportivos, escuelas, iglesia, etcétera.

En 1970, la participación de los grupos antes mencionados baja a un $31.8 \%$ manteniendo para 1975 la misma tendencia, esto debido principalmente a la llegada de una de las instituciones culturales nacionales. ${ }^{12}$

\section{b) Asociaciones consumidoras de arte}

Por su parte, los organismos no gubernamentales dedicados abiertamente a las actividades culturales bajan su presencia a un $14 \%$, debido principalmente a que disminuye la promoción por parte de las asociaciones consumidoras de arte, como el Ateneo Fronterizo, la Sociedad Manuel M. Ponce y la Asociación de Conciertos de la Comunidad de Ciudad Juárez. En el caso de las dos últimas, desaparecieron debido a la falta de apoyo oficial y a causas de elementos macroeconómicos que afectaron a la frontera norte. Lo anterior queda ejemplificado con la siguiente declaración de uno de sus miembros:

...Fíjese que la Asociación de Conciertos de la Comunidad no tenía ningún tipo de apoyo, la que nos animó a continuar fue la Asociación de El Paso, Texas. También nos financiabamos con la venta de abonos para asistir a los eventos, aunque muchas de las veces sólo lograbamos pagar a los artístas y conseguíamos prestada alguna sala para la presentación. Todo estuvo bien hasta que se vino la primer devaluación cuando subió el dólar de 12.50 a 25 pesos, y ahí se acabó la Asociación de Conciertos de la Comunidad... (Entrevista al Sr. Aquiles Valdés miembro de la Asociación de Conciertos de la Comunidad).

12 INBA, IMSS, ISSSTE, UACH, UAC, ITCJ. 


\section{c) Federación}

La federacion, como ya se mencion 6 anteriormente, aparece en este periodo como el principal promotor representando el $42 \%$, esto debido principalmente a la construcción del Teatro de la Asegurada del IMSS, del Teatro Benito Juárez y del Museo Regional de Arte e Historia; este último actualmente depende del Instituto Nacional de Bellas Artes.

Para 1960, Ciudad Juárez tenía una población de cerca de 300,000 habitantes, y a nivel nacional era una de las diez ciudades más grandes del país, por lo que demandaba mayor atención de las autoridades encargadas de la política cultural del país.

\section{d) Productores}

En este año también se da una importante participación de productores (por ejemplo: pintores, cantantes), quienes promovieron el $13.6 \%$ de los eventos a través del Jardín del Arte, grupo vinculado al Museo Regional de Arte e Historia. Es importante mencionar que a partir de este año se observa con mayor claridad la formación de grupos de artistas de diferentes especialidades, como es el caso del Jardín del Arte formado por pintores, grupos de teatro y de danza clásica.

En este periodo encontramos que hasta 1965 el Estado, en su nivel federal, se había mostrado totalmente ausente de la promocion artística. Pero en 1970 hace su aparición generando el 23\% de las actividades artísticas a través del IMSS y del Museo Regional de Arte e Historia. Este último se fundo en 1964 como parte de las obras del Programa Nacional Fronterizo (PRONAF) ${ }^{13}$ y dependía del Instituto Nacional de Bellas Artes, institución que promovió el mayor número de eventos durante este periodo.

Para 1975 el Estado, en su nivel federal, aumenta notoriamente su participación a un 63\%. Asimismo, en esta etapa la Universidad de Ciudad Juárez aparece en forma incipiente como organizadora de eventos artísticos.

Como se observ6 anteriormente, en este periodo se manifiestan tres cambios importantes; primero, los grupos ocasionales y las asociaciones consumidoras de arte mantienen su promoción, aunque su participación relativa disminuye; segundo, el municipio y el gobierno federal

\footnotetext{
${ }^{13}$ El Programa Nacional Fronterizo (PRONAF) surgió en 1964 con la intención de mejorar la imagen urbana y fomentar las actividades turísticas en la ciudad, por lo que se construyeron una serie de edificios y avenidas. Entre ellos se encuentran el Museo Regional de Arte e Historia, tiendas de artesanías, hoteles y restaurantes.
} 
incrementan su participación y, por último, aparece la Universidad Autonoma de Ciudad Juárez (UACJ) como promotor cultural.

Es importante mencionar que en esta etapa se inició la institucionalización de las actividades artísticas en Ciudad Juárez, al incrementarse la presencia de organismos gubernamentales promotores de la cultura como lo son: el Museo Regional de Arte e Historia, la UACJ, el IMSS, entre otros.

\section{Periodo 1985-1990}

Durante el periodo 1985-1990, del total de eventos promovidos, un $42 \%$ le correspondió al gobierno federal, el $28 \%$ a la universidad, el $14 \%$ a el municipio, un $10 \%$ promotores ocasionales, y el $6 \%$ a otros grupos (véase cuadro 1).

En esta etapa se observa como disminuyen las actividades artísticas promovidas por instancias no gubernamentales, como grupos ocasionales, productores y asociaciones consumidoras. Esto a consecuencia principalmente de los efectos de la crisis económica que vive el país y al surgimiento de instituciones gubernamentales a raíz de una mayor demanda de actividades artísticas y culturales por parte de algunos sectores de la sociedad.

\section{a) Promotores ocasionales}

En este periodo encontramos que para 1985, los promotores ocasionales bajaron su participación en forma muy drástica a $17.7 \%$. Pero en 1990 , éstos incrementan su presencia organizando el $23 \%$ de las actividades artísticas.

En esta misma época, destaca el incremento en la participación de las universidades $^{14}$ de la localidad, promoviendo el $28 \%$ del total de los eventos.

\section{b) Federación}

La federación se mantiene como el principal organizador al representar el $42 \%$ de los eventos. En este periodo surge la presencia del Programa Cultural de las Fronteras , institución promotora de la federación, quien junto con el gobierno del estado, el municipio e instituciones de educación, organizan el Festival de la Raza.

${ }^{14}$ Las instituciones que forman parte de este grupo son la Universidad Autónoma de Ciudad Juárez (UACJ), Universidad Autónoma de Chihuahua (UACH), Tecnológico de Ciudad Juárez, Instituto Tecnológico de Monterrey Campus Ciudad Juárez y la Escuela Superior de Agricultura Hermanos Escobar (ESAHE). 
En 1990, la participación federal muestra un declive comparativamente a la de 1980 , y en 1985 intervino en el $24 \%$ de las presentaciones. El nivel municipal eleva su presencia a un $20 \%$ debido a su participación en la organización del Festival Internacional de la Raza, en coordinación con el gobierno del estado y el Programa Cultural de las Fronteras.

En suma, la promoción de las actividades artísticas en el caso de Ciudad Juárez, está fuertemente influenciada en lo que podríamos llamar un mecenazgo liberal, cuyo mecenas en esta actividad promocional durante el periodo 1945-1990 fue principalmente el Estado en su nivel federal, seguido del municipal y finalmente el gobierno del estado con una menor participación. En esta actividad también es básico el papel que desempeñan los organismos e instituciones no propiamente culturales pero que son promotores ocasionales de las actividades artísticas, y que van desde las Logias Masónicas, clubes de servicio social (mucha de su actividad con fines de beneficencia), los medios de comunicación, principalmente la radio, hasta medianas e importantes empresas de la localidad y la iglesia, así como asociaciones abiertamente dedicadas a la promoción de la cultura, quienes, como algunos estudiosos han señalado "declaran apoyar a los creadores sin más motivos que su generosidad y sin otro fin que el de impulsar el desarrollo espiritual"' (García, 1987:28).

\section{EVOLUCIÓN DE LAS ESPECIALIDADES ARTÍSTICAS EN CIUDAD JUÁREZ, CHIHUAHUA, 1945-1990}

Como parte de la reconstrucción de la organización y promoción de las actividades artísticas en Ciudad Juárez, se usó la variable especialidades artísticas, que nos permitirá conocer qué tipo de disciplinas artísticas eran las más promovidas.

\section{Periodo 1945-1960}

Tan escaza fue la promoción de las actividades artísticas que en estos quince años encontramos que se promovieron 82 eventos, de los cuales el $15 \%$ correspondieron a danza, $46 \%$ a música, $13 \%$ a canto, $15 \%$ a teatro y $7 \%$ a literatura, como se observa en el cuadro 2.

La disciplina que tuvo una mayor participación en este periodo fue la música, esto se debió al tipo de promoción que hubo durante esos af́os. Ya que, como se menciono en la primera parte del trabajo, los grupos ocasionales fueron los que tuvieron en sus manos la organización de las actividades artísticas en esa época y la mayor parte de ellas eran del tipo festival escolar. 
CUADRO 2. Número de eventos promovidos por especialidad artística y periodos en Ciudad Juárez, Chihuahua, 1945-1990 (porcentajes).

\begin{tabular}{lccr}
\hline $\begin{array}{l}\text { Especialidad } \\
\text { artística }\end{array}$ & $1945-1960$ & $\begin{array}{c}\text { Periodos } \\
1965-1980\end{array}$ & $1985-1990$ \\
\hline Danza & 15 & 8 & 10 \\
Teatro & 15 & 13 & 34 \\
Canto & 13 & 11 & 5 \\
Literatura & 7 & 6 & 3 \\
Música & 46 & 21 & 15 \\
Artes plásticas & 4 & 36 & 26 \\
Cine-Arte & 100 & 5 & 7 \\
& $\mathrm{n}=82$ & $\mathrm{n}=100$ & 100 \\
& & $\mathrm{n}=121$ \\
\hline
\end{tabular}

FUENTE: Proyecto "Espacios Artísticos, Ciudad Juárez, 1992”. El Colegio de la Frontera Norte, Oficina Regional en Ciudad Juárez.

Lo anterior se confirma al hacer el cruce de la variable tipo de promotores y especialidades artísticas organizadas, en donde aparece que el $50 \%$ de los eventos realizados por grupos ocasionales fueron de música, como se observa en el cuadro 3.

Por su parte, en este periodo, las asociaciones consumidoras también tuvieron una gran participación, aunque a diferencia de los promotores ocasionales, la mayoría de sus eventos de música eran con orquestas o solistas de mayor reconocimiento ya sea a nivel nacional e internacional.

Otra de las especialidades, como la danza, tuvo una participación de un $15 \%$, el teatro $15 \%$ y el canto $13 \%$ (véase cuadro 2). Lo anterior como resultado del esquema de promoción que prevalecía durante esos años.

En 1950, el número de eventos artísticos presentados disminuyó y las principales disciplinas que se promovieron vuelven a ser la música, el teatro y el canto, aunque en este mismo año se realizaron una mayor cantidad de eventos literarios.

Para 1955 se incrementó el número de acontecimientos artísticos organizados con respecto al quinquenio anterior, así como la diversidad de los mismos. Del total de eventos localizados en este año, el $42 \%$ correspondió a conciertos de música, el $17 \%$ a recitales de canto, el $13 \%$ a las artes plásticas, el $17 \%$ a teatro, el $8 \%$ a danza y el $4 \%$ a los de literatura. 
CUADRO 3. Tipos de eventos artísticos en Ciudad Juárez, según clases de actores institucionales, 1945-1990.

\begin{tabular}{|c|c|c|c|c|c|c|}
\hline \multirow{2}{*}{$\begin{array}{l}\text { Tipos de } \\
\text { actores }\end{array}$} & \multicolumn{5}{|c|}{ Actividades artísticas } & \multirow[b]{2}{*}{$\begin{array}{c}\text { Total } \\
\text { eventos }\end{array}$} \\
\hline & $\begin{array}{c}\text { Artes } \\
\text { visuales }\end{array}$ & Teatro & Danza & Música & Literatura & \\
\hline Ocasionales & 11 & 25 & 6 & 50 & 8 & $100 n=48$ \\
\hline Individuales & 33 & 0 & 22 & 33 & 11 & $100 n=9$ \\
\hline Productores & 19 & 43 & 9 & 19 & 10 & $100 n=21$ \\
\hline Consumidores & 0 & 0 & 15 & 85 & 0 & $100 n=20$ \\
\hline Academias & 10 & 10 & 10 & 70 & 0 & $100 n=10$ \\
\hline Iglesia & 0 & 60 & 0 & 40 & 0 & $100 n=5$ \\
\hline Universidad & 18 & 31 & 13 & 38 & 0 & $100 n=39$ \\
\hline Municipio & 35 & 31 & 7 & 24 & 3 & $100 n=3$ \\
\hline Gob. Estado & 0 & 50 & 13 & 37 & 0 & $100 n=8$ \\
\hline Gob. Federal & 53 & 19 & 8 & 13 & 6 & $100 n=79$ \\
\hline
\end{tabular}

FUENTE: Proyecto "Espacios artísticos, Ciudad Juárez, Chihuahua, 1992". El Colegio de la Frontera Norte, Oficina Regional en Ciudad Juárez,

Hay que mencionar que durante muchos años Ciudad Juárez requirí de la participación de artístas de otras partes del país, además de los locales, los cuales eran invitados por asociaciones, instituciones, empresas, bancos, entre otros. Estos grupos de promotores marcaban las pautas del tipo de especialidad artística que organizaban en funcion de los intereses de sus miembros, razón por la que la diversificación de las actividades artísticas fue menor.

En 1960 se redujo el número de actividades artísticas y con ello también la diversidad de disciplinas, los conciertos de música clásica continuaron siendo los más promovidos, seguidos por el teatro y la danza. Es decir, se sigue con el mismo esquema de promoción artística que en 1945.

\section{Periodo 1965-1980}

En este periodo, del total de las actividades artísticas organizadas, el $36 \%$ de ellas fueron artes plásticas, el $21 \%$ de música, el $13 \%$ de teatro, el $11 \%$ de canto y el $8 \%$ de danza, como se observa en el cuadro 2 .

En 1965 se incrementó el número de eventos promovidos en relación con 1960 y con ello las disciplinas. En este año el 32\% fueron artes plásticas, $23 \%$ música, $14 \%$ canto y $9 \%$ danza. 
Los promotores que más organizaron las disciplinas artísticas antes mencionadas son el gobierno federal y municipal (ver cuadro 3 ).

Durante 1970 se generó un número similar de eventos que en 1965. Del total de las actividades organizadas en la ciudad, el $36 \%$ correspondi 6 a las artes plásticas, el $36 \%$ a la música, el $14 \%$ al canto, el $9 \%$ al teatro y el 5\% a la danza. La importante y continua presencia de las artes plásticas se debio a la gran cantidad de eventos organizados por el Museo Regional de Arte e Historia y el Jardín del Arte. El resto de las actividades como música, teatro y danza siguen siendo organizadas por el Ateneo Fronterizo, Asociación de Conciertos de la Comunidad, Sociedad Manuel M. Ponce y grupos independientes.

En 1975, el 35\% del total de actividades organizadas correspondio a las artes plásticas manteniéndose como la disciplina con más eventos organizados, el $15 \%$ a obras de teatro, el $15 \%$ a música, el $15 \%$ a cine de arte, el $10 \%$ a canto, el $5 \%$ a literatura y $5 \%$ a danza.

En este año hay tres hechos sobresalientes; primero, el Museo Regional de Arte e Historia por sí solo promovió más de la mitad de las actividades artísticas de la ciudad; segundo, aparece el cine-arte promovido principalmente por la Universidad Autónoma de Ciudad Juárez y por último, el resto de las especialidades fueron organizadas por grupos independientes.

En 1980, la actividad artística tuvo una ligera caída en relación con 1975 , representando las artes plásticas el $42 \%$ del total de los eventos organizados en ese mismo año. Por su parte, la música represento el $17 \%$, el teatro el $8 \%$, el canto $8 \%$ y la danza el $11 \%$; y los principales organizadores fueron el Museo Regional de Arte e Historia, organismos privados (clubes sociales y deportivos), asociaciones promotoras y consumidoras de arte.

Es importante resaltar que en este periodo, los cambios más relevantes que surgen son la disminución de los eventos de música, influidos por la disminución en la participación de grupos ocasionales y asociaciones consumidoras, así como por el arribo de las artes plásticas que son las actividades que más se promovieron. Lo anterior coincidió con la construcción del Museo Regional de Arte e Historia, en el que se realizaron principalmente exposiciones de pintura.

En el periodo 1985-1990, del total de eventos organizados, el $10 \%$ de ellos correspondio a danza, el $34 \%$ a teatro, el $26 \%$ a artes plásticas, el $15 \%$ a música, el $5 \%$ a canto, el $3 \%$ a literatura y el $7 \%$ a cine-arte (véase cuadro 2 ).

En esta etapa, entre los cambios más importantes que se manifiestan encontramos la disminución de la promoción de las artes plásticas, el incremento del teatro, la danza y el cine-arte. Estos cambios obedecen, principalmente, a la participación más continua de diferentes grupos de promotores, por ejemplo: las universidades y el Programa Cultural de las 
Fronteras, que junto a una serie de instituciones gubernamentales a nivel federal, estatal y municipal promovieron el Festival Internacional de la Raza.

En 1985 se contabilizaron 45 eventos, de los cuales el $29 \%$ correspondí a las artes plásticas, el $29 \%$ a teatro, el $13 \%$ a música, $11 \%$ a danza, $9 \%$ a cine-arte, $4 \%$ a canto y $4 \%$ a literatura. Del total de las actividades, casi la mitad de ellas fueron promovidas por el INBA, sobresaliendo las artes plásticas y el cine-arte, el porcentaje restante fue promovido por organismos independientes y las universidades de la ciudad y de Chihuahua.

En 1990 se incrementó en forma considerable el número de eventos artísticos. Del total, el 37\% fueron obras de teatro, el 34\% artes plásticas, el $16 \%$ música, el $9 \%$ danza, el $5 \%$ canto, el $6 \%$ cine y el $3 \%$ literatura.

En este periodo es importante destacar la presencia de una serie de instituciones; primero, las universidades y segundo, el Programa Cultural de las Fronteras, que en forma conjunta con otras instituciones organizan el Festival Internacional de la Raza y que dan la idea de una mayor coordinación en la promoción artística en la ciudad.

\section{ORGANIZACIÓN DE LOS ESPACIOS FÍSICOS PARA LA EXPRESIÓN ARTÍSTICA}

Uno de los principales obstáculos para el desarrollo de las actividades artísticas en Ciudad Juárez ha sido la falta de infraestructura, por lo que tratamos de reconstruir qué tipos de espacios físicos se han utilizado a partir de 1945.

\section{Periodo 1945-1960}

De 1945 a 1960 el $42 \%$ de los eventos artísticos se realizaban en salones, $14 \%$ en cines, $10 \%$ en escuelas, $4 \%$ en teatros y $4 \%$ en auditorios (véase cuadro 4).

Lo anterior nos da una idea de la falta de infraestructura para el desarrollo de las actividades artísticas en esta época, por lo que los grupos promotores improvisaban los espacios, ya que la mayor parte de los eventos durante estos quince años se realizaron en salones de baile y cines, que no cuentan con el acondicionamiento necesario para el buen desarrollo de estas actividades.

De 1945 a 1965 el listado de los espacios ocasionalmente utilizados es muy amplio, y van desde las salas cinematográficas, principalmente, salones de actos de templos, centros escolares, estaciones de radio, restaurantes, residencias particulares y existe el caso de una familia de artistas 
CUADRO 4. Lugares en los cuales se realizaron eventos artísticos por periodos en Ciudad Juárez, Chihuahua, 1945-1990 (porcentajes).

\begin{tabular}{lccc}
\hline Lugares & & Periodos & \\
& $1945-1960$ & $1965-1980$ & $1985-1990$ \\
\hline Salones & 42 & 21 & 31 \\
Cines & 14 & & \\
Teatros & 4 & 14 & 18 \\
Auditorios & 4 & 2 & 18 \\
Escuelas & 10 & 2 & 1 \\
Estadios & 1 & 46 & 1 \\
Gimnasios & & 1 & 21 \\
Museos & & 5 & 3 \\
Galerías & 25 & 100 & 7 \\
Otros & 100 & $\mathrm{n}=97$ & $\mathrm{n}=104$ \\
& $\mathrm{n}=72$ & & \\
& & & \\
\hline
\end{tabular}

FUENTE: Proyecto "Espacios artísticos, Ciudad Juárez, 1992". El Colegio de la Frontera Norte, Coordinadora en Ciudad Juárez.

que construy 6 su propio teatro. ${ }^{15}$ En esta época la radio juega un papel sobresaliente al "masificar y ampliar" la difusion de las actividades artísticas, ya que las personas que no asistían físicamente a los eventos artísticos, podían escuchar los programas desde sus casas, además de que este medio daba a los programas artísticos alcance regional en algunos de los casos.

Los espacios privados más frecuentemente utilizados fueron el Salón Zaragoza, el Casino Juárez y Televicentro, donde los programas del Ateneo Fronterizo fueron trasmitidos a través del canal 5. No obstante los esfuerzos de la difusión cultural, la presencia física de espectadores se

15 La fecha de fundación es incierta, pero algunas personas señalan que a principios de 1940 , otros más, una década después. Lo cierto es que en aquellos años el Teatro Libertad fue de los pocos que sirvieron de foro por muchos años para las sesiones del Ateneo Fronterizo. Ignacio Esparza, cronista de la ciudad, señala que el teatro se mandó construir exprofeso por sus dueños (ya fallecidos): el profesor Fernando Navarro y la maestra Libertad Montelongo de Navarro para sus actividades artísticas y educativas, ya que ellos fundaron una academia de música. 
reduce principalmente a los sectores medios y altos, dado lo elitista de los eventos como lo eran los conciertos, recitales de piano, teatro y otros, a pesar de realizarse en espacios como las salas cinematográficas que son espacios más familiarizados con el sector popular. Quizá los espacios que atrajeron a un poco más de público fuera de los sectores antes citados fueron el Salon Zaragoza y Televicentro en la presentación de los programas del Ateneo Fronterizo, por su actitud más abierta a la comunidad en general, pero también se puede considerar que su tipo de construcción, así como su ubicación en la zona centro de la ciudad, hace más fácil su acceso.

\section{Periodo 1965-1980}

En el periodo 1965-1980 se manifiestan los siguientes cambios: el 46\% de los eventos artísticos se realizaron en museos o salas, $21 \%$ en salones, $14 \%$ en auditorios y $8 \%$ en escuelas, como se observa en el cuadro 4 . Este cambio está relacionado con el crecimiento de la ciudad y con ello la construcción de algunos teatros, salas y gimnasios, en donde se realizaban la mayor parte de las actividades artísticas.

En estos años, se observa una mayor presencia de eventos artísticos en recintos oficiales debido a la llegada de las instituciones culturales nacionales, los que reúnen la infraestructura mínima para un buen desarrollo de las actividades culturales.

A mediados de los sesenta y hasta los noventa, los espacios propiedad del Estado como el Museo de Arte e Historia, el Auditorio Benito Juárez, el Teatro de la Nación, la Sala de Convenciones del PRONAF o Sala de Espectáculos del INBA, son los más frecuentemente utilizados para actividades artísticas hasta en un $87 \%$.

En suma, en este periodo el uso de infraestructura artística en espacios institucionales es cada vez más frecuente.

\section{Periodo 1985-1990}

En esta etapa disminuye el uso del Museo Regional de Arte e Historia como un espacio cultural, representando el $21 \%$ de eventos realizados en el mismo, los salones vuelven a incrementar su participación alcanzando el $31 \%$, por su parte los teatros y auditorios aumentan su presencia al $18 \%$ en ambos casos. Asimismo, surgen nuevos espacios artísticos como galerías, gimnasios y estadios.

Es decir, en estos últimos cinco años hay una mayor diversificación de los espacios en donde se llevan a cabo actividades artísticas, producto de 
su crecimiento poblacional que demanda la creación de un mayor número de espacios en los que se presenten los eventos.

\section{REFLEXIONES FINALES}

El análisis de la organización y promoción de las actividades artísticas en Ciudad Juárez muestra características propias producto de su realidad fronteriza.

Durante el periodo 1945-1960, las prácticas artísticas se encuentran en un estado de baja institucionalización, la continuidad del arte está solamente dada por promotores ocasionales y asociaciones consumidoras de arte, que sobreviven durante estos años con recursos propios, y se caracterizan por su volatilidad institucional o su débil continuidad en este tipo de actividades. Por su parte, las disciplinas principalmente promovidas son: canto, música y teatro. Asimismo, los espacios físicos usados para presentar actividades artísticas muestran el mismo comportamiento anterior, es decir, son salas, cines y casas particulares no creadas técnicamente para el desarrollo de actividades artísticas, no hay apoyos económicos y no hay continuidad en su uso.

A partir de 1965 se inició la institucionalización de las actividades artísticas y culturales en Ciudad Juárez, al incrementarse la presencia de organismos gubernamentales como promotores. Lo anterior también modifico el patrón de espacialidades artísticas ampliando el espectro de las mismas, ya que se organizaron eventos de canto, danza, música, pintura, escultura, etcétera.

En lo que respecta a los espacios físicos utilizados para la realizacion de actividades artísticas, aparecen los primeros teatros y museos.

De 1985 a 1990 se mantiene una alta institucionalizacion de las actividades artísticas, sin embargo, aparecen nuevos actores sociales como las universidades y el Programa Culturas de las Fronteras. En estos últimos cinco años aparecen actividades como cine-arte y literatura, que se agregaron a otras ya existentes y anteriormente mencionadas.

Del mismo modo existe una mayor infraestructura para el desarrollo de las actividades artísticas, entre los que se encuentran gimnasios, galerías y estadios.

En general, durante los últimos treinta años la promoción de actividades artísticas en Ciudad Juárez está influenciada por lo que podríamos llamar un mecenazgo liberal, cuyo mecenas en esta actividad promocional fue el gobierno en sus tres niveles. 


\section{BIBLIOGRAFÍA}

GARCÍA Canclini N. 1987. Políticas Culturales en América Latina, Editorial Grijalbo, México, D.F.

LEÓN-PORTILLA, M. 1976. Culturas en peligro, Alianza Editorial Mexicana, México, D.F.

MAIRET, G. y Chatelet, F. 1978. Les Ideologies. Marabout, Verviers: tomo 2.

ZÚNIIGA, Víctor. 1992. "Promover el arte en una ciudad del norte de México", en: Estudios Sociológicos (en prensa).

ZÚNIIGA, Víctor. 1992 "Política cultural en la frontera norte: el Estado y la producción artística en Monterrey". (Mimeo), COLEF. 